\title{
Seeded Watersheds for Combined Segmentation and Tracking of Cells
}

\author{
Amalka Pinidiyaarachchi ${ }^{1,2, \star}$ and Carolina Wählby ${ }^{1}$ \\ 1 Centre for Image Analysis, Uppsala University, Sweden \\ 2 Dept. of Statistics and Computer Science, University of Peradeniya, Sri Lanka \\ amalka@cb.uu.se, carolina@cb.uu.se
}

\begin{abstract}
Watersheds are very powerful for image segmentation, and seeded watersheds have shown to be useful for object detection in images of cells in vitro. This paper shows that if cells are imaged over time, segmentation results from a previous time frame can be used as seeds for watershed segmentation of the current time frame. The seeds from the previous frame are combined with morphological seeds from the current frame, and over-segmentation is reduced by rule-based merging, propagating labels from one time-frame to the next. Thus, watershed segmentation is used for segmentation as well as tracking of cells over time. The described algorithm was tested on neural stem/progenitor cells imaged using time-lapse microscopy. Tracking results agreed to $71 \%$ to manual tracking results. The results were also compared to tracking based on solving the assignment problem using a modified version of the auction algorithm.
\end{abstract}

\section{Introduction}

Cell migration analysis is of interest in many different biological applications, e.g. when studying leucocytes, fibroblasts, or cancer cells [123]. This paper is part of a greater project with the aim to aid in the investigation of the mechanisms behind differentiation of neural stem/progenitor cells by creating a system for analyzing the motion of such cells in vitro, previously described in [4]. Once the cells have been imaged using time-lapse microscopy, the individual cells in each image have to be automatically segmented and tracked. Cells in tissue culture are non-rigid, irregularly shaped bodies. As the cells move, grow and divide they take on a variety of different shapes and the contrast between the cell boundary and the image background varies continually. Several techniques for cell image segmentation have been proposed in the recent years. A simple and popular method is thresholding, based on histogram characteristics of the pixel/voxel intensities of the image [5]. However, transition between object and background may be diffuse, making an optimal threshold level, and thereby an accurate description of the cellular shape, difficult to find. In addition, thresholding techniques do not guarantee closed object contours, and often require

\footnotetext{
* The first author was funded by the SIDA-IT project.
} 
substantial post-processing [10:11. Instead of defining the border between object and background by a threshold, similarity in intensity, gradient, or variance of neighboring pixels can be used. This is usually referred to as region growing, and includes techniques such as watershed segmentation [1213]. Watershed segmentation often results in over-segmentation due to intensity variations within both objects and background. Seeded watersheds, where the number of segmentation boundaries is limited to a set of input seeding regions, have been used for segmentation of cell nuclei in images produced by fluorescence microscopy 14. Fully automatic seeding often results in more than one seed per object, or objects containing no seed at all. Over-segmentation caused by extensive seeding can however be reduced by model-based region merging [15].

To improve the segmentation, the segmentation and tracking can be combined. A popular method that combines the segmentation and tracking is active contours (snakes), previously used for cell segmentation/tracking in e.g. 116 17. A significant drawback with the traditional snake is its inability to deal with splitting cells. Another technique combining segmentation and tracking is demonstrated in [2], where the directional movement of cells induced by a direct current (galvanotaxis) is studied. Standard thresholding combined with clustering results in a coarse segmentation, which is refined further using the result of an association/tracking algorithm. Tracking is done using a modified version of the Kalman filter. This method works well because of the known directional movement of the cells. However, assumptions about a cells state equations are potentially risky in cases where little is known about the laws governing the cell motion, and when the purpose of the analysis is to gain more information about cell kinematics. Other common tracking techniques are the nearest-neighbor method [18] and correlation matching [6]. Nearest-neighbor techniques require low cell density and high temporal sampling rate, while correlation matching instead requires the individual cells features, such as size, shape and intensity, to be fairly constant between two consecutive frames, which is not often the case for cells.

This paper presents a combined segmentation and tracking method based on seeded watersheds. The presented technique is compared to manual tracking results as well as with a previous approach using a combination of nearestneighbor and correlation matching techniques where the cells are associated using the auction algorithm 47 .

\section{The Imaging System}

The time-lapse microscopy and imaging system has previously been described in [4, and will only be discussed briefly. For cell tracking experiments, cells were cultured in a microscope incubator and imaged every 10 minutes for 45.5 hours. Time-lapse microscopy of neural stem/progenitor cells in vitro requires a sterile environment and a feedback control of temperature and $\mathrm{pH}$. This was achieved using a closed incubation chamber, a heater unit and warm, filtered air together with a $\mathrm{CO}_{2}$ mixture (regulating the $\mathrm{pH}$ ) circulating within the acrylic chamber, all regulated by a controller unit. An inverted light microscope with 

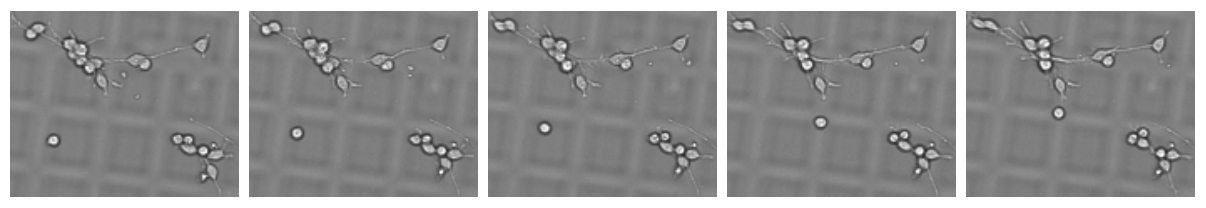

Fig. 1. Cut-outs from five consecutive time frames, each 10 minutes apart. As can be seen, some cells are fairly stationary, while others move faster.

a 20X ordinary bright field objective was used for imaging. No special contrast method such as phase contrast, or DIC, was used. By slight defocusing, higher contrast is traded for lower resolution. Images were captured by a CCD video camera and the specimen was moved in the horizontal plane by a motorized microscope stage to image the separate wells. A focus stack was captured by sweeping the approximate interval, and the image with the best contrast was selected by an auto-focus function searching for the optimal focus position based on local image contrast. A slight image drift in both $\mathrm{x}$ and $\mathrm{y}$ direction between consecutive image frames was corrected by estimation of the translation for each pair of frames using the correlation in the Fourier domain. The images used in this paper were 30 different image sequences acquired at two different occasions. Each image sequence contained 273 gray scale frames, each of size 634 by 504 pixels. A series of cut-outs from five consecutive time frames is shown in Fig. 1.

\section{Segmentation and Tracking}

Cell segmentation and tracking over time is performed as a combined procedure.

Initial Seeding. The image segmentation is based on seeded watershed segmentation. First, seeds representing objects (cells) and background are defined. As the image intensities of the cells show a range of values partly overlapping with the background intensities, see Fig. [2(a), intensity thresholding will not separate cells from background in a satisfactory way. A more effective method for separating objects from background is using variance measures [10. A 10 by 10 variance filter is applied to the image, and the image background seed is defined by a fixed intensity threshold $(t=300)$ in the variance image, shown in Fig. $2(b)$. The resulting background seed is shown in gray in Fig. 2(c). Object seeds at the initial step are found using the extended h-maxima transform [8] that filters out local maxima using a contrast criterion. All maxima with heights smaller than a threshold level h are suppressed. A low h will result in many seeds, often more than one seed per cell, while a high h will leave some cells without a seed. Since the subsequent merging step (described below) reduces over-segmentation due to extensive seeding, a comparably low h value $(\mathrm{h}=16)$ produces a suitable seed. All foreground seeds are uniquely labeled using connected component labeling. The seeds obtained from the h-maxima transform (referred to as h-maxima seeds hereafter) are then combined with the background seed identified by the variance 
thresholding. The background seed is assigned the highest label after labeling of object seeds. Object (white) and background seeds (gray) are shown in Fig. 2(c).

Watershed segmentation. The combined object and background seed information is used as input for watershed segmentation. Seeded watershed segmentation is applied to the inverse of the original intensity image. The dark edges of the cells are thus interpreted as ridges, and the brighter cells and background as shallow valleys in the watershed landscape. Seeds represent holes in the landscape, and as the landscape is submerged in water, the water will start to flow into the minima of the landscape, creating one catchment basin associated with each local minima. As the water rises, water from neighboring catchment basins will meet. At every point where two catchment basins meet, a dam, or watershed is built. These watersheds are the segmentation of the image. The speed of watershed segmentation is improved using sorted pixel lists 9 . The initial result of seeded watershed segmentation of Fig. 2(a) using the seeds from Fig. 2(c) is shown in Fig. 2(d).

Merging. Watershed segmentation often results in over-segmentation. The segmentation is therefore followed by two merging steps. The first merging step removes extra regions due to non-seeded local minima. These regions are merged with the neighboring region towards which it has its weakest border. The weakest border is defined as the border in which the mean intensity of the inverse of the original image is the least 15. The seeded neighbor may be either object or background, and the merging continues until all non-seeded objects are merged. See the result of the first merging step in Fig. 2(e). The second merging step deals with over-segmentation resulting from extensive h-maxima seeding. This over-segmentation is reduced by removing region boundaries crossing bright parts of the image, e.g., a boundary dividing a bright cell in two. In this case we continue the merging until all remaining objects have a defined maximum average intensity along the border $(t=100)$. This step will not only reduce oversegmentation, but also merge false objects, such as debris, with the background. The final segmentation result is shown in Fig 2(f).

Tracking by propagation of seeds. The resulting segmentation is then processed to obtain seeds that are used for tracking the objects over time. The centroid of each region is computed and these centroids are then dilated with a 3 by 3 structuring element. These seeds (referred to as centroid seeds hereafter) are labeled according to the preceding segmentation. Each centroid seed is compared with the h-maxima seeds of the next frame to identify any overlap between the two seeds. The most frequent value of the overlap is chosen when updating the label values of the h-maxima seeded frame. H-maxima seeds that do not overlap with centroid seeds keep their original label, and centroid seeds that do not overlap with h-maxima are transferred directly to the new set of seeds. In order to make the check efficient, initial labeling of the h-maxima seeds are adjusted to take the values starting from the highest value of the labels from the previous frame. This enables an easy implementation of the checking process with the trade off of label values increasing fast if no overlap is found. The 


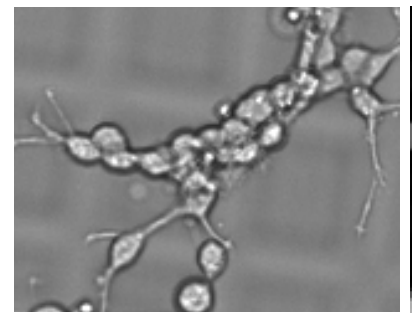

(a)

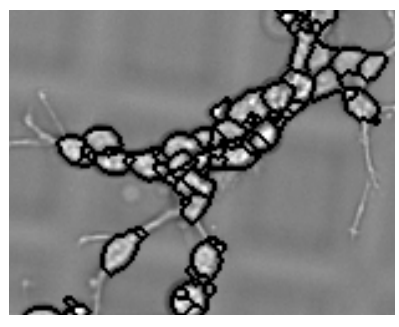

(d)

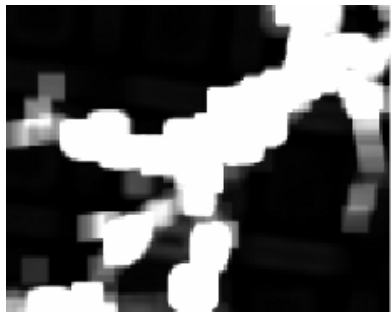

(b)

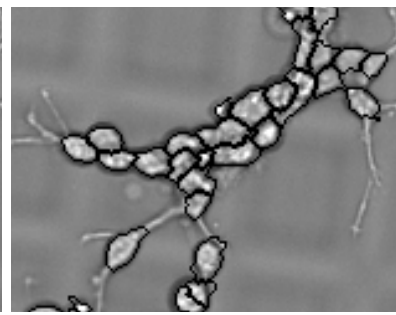

(e)

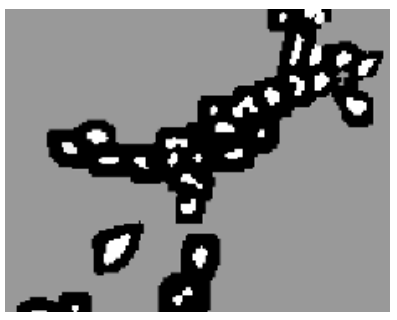

(c)

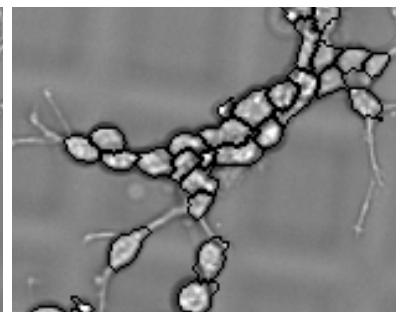

(f)

Fig. 2. The steps of the segmentation algorithm. (a) Original image. (b) Variance image. (c) Foreground seeds (white) and background seeds (gray). (d) Result of initial seeded watershed segmentation. (e) Result after merging to remove non-seeded objects. (f) Final result after second merging to remove over-segmented objects.

combined set of seeds together with the background seed is thereafter used as input for watershed segmentation followed by the merging steps described above. This yields a result where cells inherit their labels from the previous frame if a correspondence is found or are assigned a new unique label if no correspondence is found.

Implementation. The segmentation and tracking method was prototyped using Matlab (The MathWorks, Inc.) together with the Image Processing Toolbox and some functions from the DipImage toolbox (The Pattern Recognition Group of the TU, Delft).

\section{Results}

Validation of automatic segmentation and tracking demands image sequences of correctly tracked cells to which the automatic tracking results can be compared. Generation of ground truth for large image sequences is a very time consuming task as it has to be done by manual inspection of each image frame. A userfriendly graphical application for manual correction of segmentation and tracking results described in 4 was used and segmentation errors were detected by 


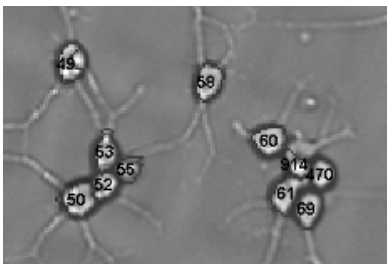

(a)

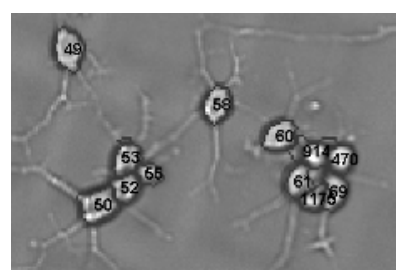

(d)

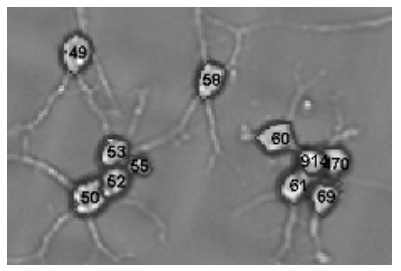

(b)

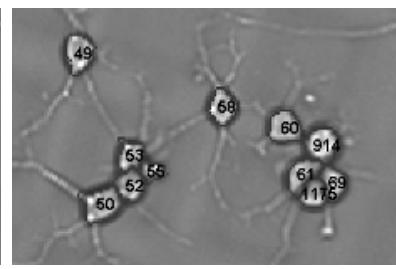

(e)

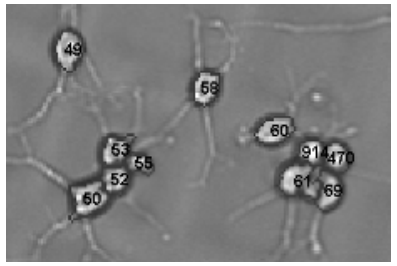

(c)

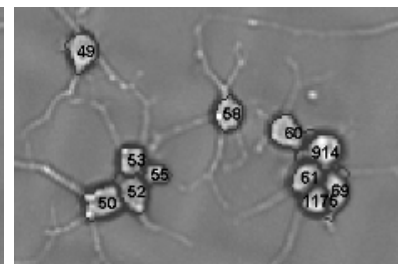

(f)

Fig. 3. The segmentation and tracking result (represented by a number printed on each cell) of 6 consecutive time frames. The numbers change when cells split (cell 61 becomes 61 and 1175 in d) and merge (914 and 470 become 914 in e).

pairwise comparison of automatically segmented and tracked frames and manually corrected ground truth. Four types of segmentation errors were identified: over-segmented cells, under-segmented cells, partially detected cells, and missed cells. Tracking errors were defined as changes of labels between consecutive image frames. If a single cell changes label once during a 100 -frame sequence, it is considered as a $1 \%$ error. As the same cell can be correctly segmented but incorrectly tracked between two image frames, or incorrectly segmented but correctly tracked, we count tracking errors only in frames that are correctly segmented.

A total of 185 cells were chosen for manual tracking based on the result of an immunostaining performed after the completed time-lapse experiment [4]. Tracks represent a fairly random selection of cells available in the final image of each time-lapse experiment. Images were acquired every 10 minutes for 45.5 hours. During this comparatively long observation time, cells can move long distances, and disappear from the field of view. The cells also divide, and group in clusters where they can no longer be tracked, not even by manual inspection. Therefore, only $28 \%$ of the cells chosen for manual correction were present for the full 45.5 hour experiment. The total number of cells that were correctly segmented and tracked over time divided by the total number of tracked cells over time was $71.3 \%$. The corresponding error rate for tracking using our previous approach based on a combination of nearest-neighbor and correlation matching techniques was $88 \%$. The difference in corrections made by two different users correcting the same sequence, i.e. inter-observer variability, was estimated to $2.5 \%$. This can be used as a measure of the variance of the error rate of the corrected sequences. A series of tracking results is shown in Fig. 3 . 


\section{Discussion}

This paper presents a combined segmentation and tracking method based on seeded watersheds and rule-based merging. It allows for object splitting, addition of new objects, and disappearance of objects. It does however fail if the objects move more than one object radius between consecutive frames. The method was tested the complex problem of analyzing cell migration on the basis of light microscopy image sequences. Cells sometimes congregate, forming large cell clusters. In these clusters, individual cells are difficult, or even impossible to resolve. This causes problems both for the segmentation and tracking. Unfortunately, we have found that growing the cells at a lower density is not a solution, since the cells are more likely to die if seeded to sparsely. The error counting method differs significantly from that of Zimmer et al. [16] that requires a cell to be correctly tracked from the first frame to the last, or it is counted as an error. The time interval also differs from $1 \mathrm{~s}$ between two frames in their method to 10 minutes in ours giving a completely different type of image sequences to work with. The advantage of this method is its simplicity in implementation and the ability to perform both segmentation and tracking together. The method could be used very effectively for tracking objects when there is less clustering and less rapid motion than in the complex set of images we have tested it on. This can also be used as the first step to obtain a result that is used as the input to a more detailed method that addresses such complexities. The fact that the simple steps used in order to segment and track the objects giving an encouraging result proves that, with further improvements it can become as effective as any other similar method.One such improvement will be to have further dilation on the centroid seeds in cases a correspondence could not be found.The current implementation takes a few hours to run on an image sequence of about two hundred cells and 250 time-frames. Further studies will be done with the intention of reducing the processing time of large sequence of images in Matlab by combining its capabilities with other environments such as $\mathrm{C} / \mathrm{C}++$.

Acknowledgments. The authors would like to thank K. Althoff, J. Faijerson, J. Degerman and T. Gustavsson for providing image data.

\section{References}

1. F. Leymarie and M. Levine: Tracking deformable objects in the plane using an active contour model, IEEE Trans. Pattern Anal. Machine Intell., 15(6) 617-634 (1993)

2. T. Kirubarajan, Y. Bar-Shalom, and K. Pattipati: Multiassignment for tracking a large number of overlapping objects, IEEE Trans. Aerosp. Electron. Syst., 37(1) 2-21 (2001)

3. P. Umesh Adiga and S. Chaudhuri: Segmentation of volumetric histopathological images by surface following using constrained snakes, in Proc. of 14th Int. Conf. Pattern Recogn., 2 1674-1676 (1998) 
4. K. Althoff, C. Wählby, J. Faijerson, J. Degerman, A. Pinidiyaarachchi, M. Gedda, P. Karlsson, T. Olsson, P. Eriksson, E. Bengtsson, T. Thorlin, and T. Gustavsson: Time-lapse microscopy and image analysis for in vitro cell migration analysis. (submitted)

5. P. Sahoo, S. Soltani, A. Wong, and Y. Chen: A survey of thresholding techniques, Comp. Vis. Graph. Im. Proc., 41 233-260 (1988)

6. T. Gustavsson, K. Althoff, J. Degerman, T. Olsson, A.-C. Thoreson, T. Thorlin, and P. Eriksson: Time-lapse microscopy and image processing for stem cell research modeling cell migration, in Medical Imaging 2003: Image Processing, 5032 1-15 (2003)

7. D. Bertsekas: Auction algorithms, in Linear Network Optimization: Algorithms and Codes, pp. 167-244, The MIT Press, 1 ed. (1991)

8. P. Soille, Morphological Image Analysis: Principles and Applications. SpringerVerlag (1999)

9. L. Vincent and P. Soille: Watersheds in digital spaces: an efficient algorithm based on immersion simulations, IEEE Trans. Pattern Anal. Machine Intell., 13(6) 583598 (1991)

10. K. Wu, D. Gauthier, and M. Levine: Live cell image segmentation, IEEE Trans. Biomed. Eng., 42(1) 1-12 (1995)

11. C. Ortiz de Solorzano, E. Garcia Rodriguez, A. Jones, D. Pinkel, J. Gray, D. Sudar, and S. Lockett: Segmentation of confocal microscope images of cell nuclei in thick tissue sections, Journal of Microscopy, 193 212-226 (1999)

12. S. Beucher and C. Lantuéjoul: Use of watersheds in contour detection, in Int. Workshop on Image Processing, CCETT, Rennes, France (1979)

13. L. Vincent: Morphological grayscale reconstruction in image analysis: applications and efficient algorithms, IEEE Trans. Image Processing, 2(2) 176-201 (1993)

14. G. Landini and E. Othman: Estimation of tissue layer level by sequential morphological reconstruction, Journal of Microscopy, 209(2) 118-125 (2003)

15. C. Wählby, I.-M. Sintorn, F. Erlandsson, G. Borgefors, and E. Bengtsson: Combining intensity, edge, and shape information for $2 \mathrm{D}$ and $3 \mathrm{D}$ segmentation of cell nuclei in tissue sections, Journal of Microscopy, 215(1) 67-76 (2004)

16. C. Zimmer, E. Labruyere, V. Meas-Yedid, N. Guillen, and J.-C. Olivo- Marin: Segmentation and tracking of migrating cells in videomicroscopy with parametric active contours: a tool for cell-based drug testing, IEEE Trans. Med. Imag., 21(10) 1212-1221 (2002)

17. O. Debeir, I. Camby, R. Kiss, P. Van Ham, and C. Decaestecker: A model-based approach for automated in vitro cell tracking and chemotaxis analyses, Cytometry, 60A 29-40 (2004)

18. Z. Demou and L. McIntire: Fully automated three-dimensional tracking of cancer cells in collagen gels: Determination of motility phenotypes at the cellular level, Cancer Research, 62 5301-5307 (2002) 